\title{
Análisis crítico de un antimicrobiano sub-óptimo, de frecuente sobre-utilización e inadecuada dosificación: Vancomicina
}

\author{
Marcelo Wolff1,2,3,4, Raúl Quintanilla ${ }^{1,2,4,5}$, Juan Pablo Carrasco ${ }^{1,6}$ y Marcela Cifuentes ${ }^{7}$
}

\section{Critical review of a suboptimal antibiotic, frequently overused and inappropriately dosed: Vancomycin}

Background: Vancomycin, standard parenteral therapy for Gram positive cocci resistant to traditional beta-lactam antibiotics (Staphylococcus aureus and coagulase negative staphylococci [CNS]) and Enterococcus spp, frequent agents of nosocomial infections, is extensively used empirically in that setting. However, its pharmacological weakness, narrow therapeutic margin and poorly predictable pharmacokinetics, make it a suboptimal drug according to contemporary criteria. Vancomycin is over utilized due to both, overestimation of bacterial infections and, in genuine cases, overestimation of the etiological role of these resistant cocci, either nosocomially or community acquired. New threats narrow further its therapeutic role: poorer outcomes in infections with higher vancomycin MIC and resistance by enterococci. It is frequently given at inappropriate dosage and intervals, failing to: give loading dose when recommended, measure blood levels, adjust dosing to changing renal function and continued use when not necessary. Newer anti staphylococcal drugs haven't replaced completely the role of vancomycin, which maintains its usefulness in contemporary medicine. Conclusion: Understanding the strengths and weaknesses of vancomycin, current epidemiology and microbiology of infections for which it may be indicated, as well as the proper administration and monitoring, together with a prudent and selective indication will allow to preserve its present and future utility in the changing medical scenario.

Keywords: Vancomycin; methicillin resistant $S$. aureus; antibiotic stewardship; misuse of antibiotics.

Palabras clave: Vancomicina; uso racional de antimicrobianos; Staphylococcus aureus resistente a meticilina.

\section{Introducción y consideraciones generales}

$\mathrm{V}$ ancomicina es un antimicrobiano antiguo, aprobado en 1958 en Estados Unidos de América (E.U.A.), de acción fundamentalmente antiestafilocócica. Tuvo un progresivo descenso en su uso por toxicidad de las formulaciones iniciales, administración exclusiva endovenosa y el desarrollo de fármacos anti-estafilocócicos más potentes, seguros, versátiles $\mathrm{y}$ de mayor margen terapéutico. Su necesidad de uso se recupera y expande en la década de 1990 frente al surgimiento y diseminación de cepas de Staphylococcus aureus resistentes a esas moléculas más activas ( $S$. aureus resistente a meticilina [SARM]), la pérdida de actividad de penicilina y ampicilina frente a Enterococcus spp, así como la aparición de Staphylococcus coagulasa negativa (SCN), de menor e incierta susceptibilidad a $\beta$-lactámicos anti-estafilocócicos, como agente patógeno oportunista en hospederos inmunodeprimidos o pacientes debilitados. Las cepas descritas con este mayor perfil de resistencia, son fundamentalmente patógenos responsables de las hoy en día denominadas infecciones asociadas a la atención de salud (IAAS), de persistente ocurrencia dada la mayor vulnerabilidad y susceptibilidad a infecciones de la población atendida actualmente (mayor sobrevida de pacientes con patologías graves, inmunosupresión, cirugías más complejas, mayor portación de elementos protésicos, así como envejecimiento global de la población). Frente a la sospecha o documentación de una IAAS en esta población, el estándar terapéutico es el inicio precoz de una terapia antimicrobiana empírica o definitiva luego de, idealmente, tomar las medidas diagnósticas adecuadas. Vancomicina es, sin duda, el antimicrobiano más frecuentemente usado en esta situación para otorgar cobertura antibacteriana contra los agentes mencionados, especialmente en unidades de pacientes críticos ${ }^{1}$. A menudo, este antimicrobiano se asocia a otros, de acción anti bacilos gramnegativos, en una terapia empírica inicial para ampliar la cobertura antimicrobiana.

A nivel nacional, en el año 2013, el uso de vancomicina en unidades críticas, expresado en dosis diarias definidas (DDD) era igual a 21,8 ; esto significa que aproximadamente 22 pacientes al día estaban siendo tratados con vancomicina por cada 100 camas ocupadas ${ }^{2}$. Datos más recientes del Grupo Colaborativo de Resistencia Bacteriana (GCRB) de Chile mostraron para el año 2017 una

\author{
'Hospital San Borja Arriarán \\ ${ }^{2}$ Unidad de Infectología de \\ adultos. \\ ${ }^{3}$ Facultad de Medicina, \\ Universidad de Chile. \\ ${ }^{4}$ Fundación Arriarán \\ ${ }^{5}$ Comité de Infecciones Asociadas \\ a la Atención de Salud. \\ ${ }^{6}$ Servicio de Farmacia. \\ ${ }^{7}$ Hospital Clínico Universidad \\ de Chile. \\ Los autores declaran ausencia de \\ conflictos de interés.
}

Recibido: 10 de febrero de 2019 Aceptado: 29 de octubre de 2019

Correspondencia a: Marcelo Wolff Reyes marcewolff@yahoo.com 
DDD de 16 en un total de 28 unidades críticas (GCRB, datos no publicados). En el servicio de medicina del hospital de los autores, $45 \%$ de las terapias empíricas por sospecha de IAAS incluyen vancomicina (J P Carrasco, datos no publicados). A pesar de este uso masivo, se ha cuestionado la utilidad actual de vancomicina, tanto por su real efectividad y seguridad, como por su frecuente inadecuada indicación y dosificación ${ }^{3}$. No obstante su extensivo uso empírico en el ambiente hospitalario, tan sólo en una proporción muy minoritaria se documenta su real necesidad ${ }^{4}$, y más aún, en neumonía nosocomial no se han documentado desenlaces adversos en los pacientes cuando se suspende vancomicina al no comprobarse presencia de agentes etiológicos en los cuales es de primera elección ${ }^{5}$.

Esta revisión tiene como objetivo hacer un análisis crítico del fármaco, precisar sus fortalezas, debilidades y administración inadecuada, para optimizar su uso pues, a pesar de sus inconvenientes, sigue teniendo vigencia cuando se usa apropiadamente. Para revisar estos aspectos se analizan características de la molécula, de los patógenos susceptibles y las infecciones que causan (nosocomiales o comunitarias) y su contexto epidemiológico, consideraciones fármaco-terapéuticas generales, los resultados terapéuticos más específicos, los errores más frecuentes en su uso y algunas recomendaciones para evitarlos. Las consideraciones aquí señaladas, especialmente en el campo epidemiológico y clínico, se aplican fundamentalmente a la práctica de medicina de adultos $\mathrm{y}$, aunque se aportan datos objetivos de respaldo, incorporan un componente importante de opiniones personales de los autores, sujetas a visiones distintas.

\section{El antimicrobiano}

Vancomicina es un antimicrobiano de la familia glicopéptidos, de estructura compleja, cuyo mecanismo de acción es la inhibición de etapas tardías de la síntesis de la pared bacteriana durante la replicación activa. Tiene actividad contra microorganismos grampositivos, en particular especies de Staphylococcus (S. aureus,
Staphylococcus epidermidis y otros SCN), Streptococcus pyogenes, Streptococcus pneumoniae y Streptococcus agalactiae, entre otros. Tiene actividad (bacteriostática) sobre la mayoría de las cepas de Enterococcus faecalis $(95 \%)$, y sólo sobre una pequeña fracción de las cepas de Enterococcus faecium $(<20 \%)$. También es activa contra Clostridium spp (incluido $C$. difficile, actualmente denominado Clostridioides difficile), Listeria spp y Corynebacterium spp. Además, la mayoría de las cocáceas grampositivas anaerobias son susceptibles ${ }^{6}$. El Clinical Laboratory Standards Institute (CLSI) de E.U.A., referente nacional tradicional para los puntos de corte de susceptibilidad bacteriana, recomienda que la susceptibilidad de Staphylococcus spp a vancomicina se determine por concentración inhibitoria mínima (CIM), ya sea por estudio de microdilución o por gradiente de concentración en agar (epsilometría-E test), este último de menor uso actual debido a su tendencia a sobre-estimar la CIM. El método de difusión por sensidisco tradicional no diferencia entre cepas de $S$. aureus sensibles, de intermedias a vancomicina ni entre sensibles, intermedias y resistentes en SCN. Para vancomicina los puntos de corte recomendados en su versión de 2018 se muestran en la Tabla $1^{7}$. Para Enterococcus spp, en cambio, se mantiene la posibilidad de testear la susceptibilidad con el método de difusión en discos con lectura a las $24 \mathrm{hrs}$. de incubación. Los puntos de corte del CLSI para este género se muestran en la Tabla 1.

Adicionalmente, en Chile se han ido incorporando últimamente las recomendaciones del Comité Europeo de Testeo de Susceptibilidad Antimicrobiana (EUCAST) que en algunos casos difieren de los del CLSI y que podrían tener mejor correlación con la respuesta clínica. En 2018, dado que con valores de CIM de vancomicina de $\geq 2 \mu \mathrm{g} / \mathrm{mL}$ las cepas de $S$. aureus se encuentran en el límite de la distribución de tipo silvestre ${ }^{8}$ y pudiera haber una respuesta clínica disminuida ${ }^{9}$, se ha propuesto que el punto de corte para resistencia se reduzca a $>2 \mu \mathrm{g} /$ $\mathrm{mL}$ eliminando la categoría de $S$. aureus de sensibilidad intermedia a vancomicina, ya que las infecciones graves con esa cepas no se pueden tratar con dosis aumentadas de

\begin{tabular}{|c|c|c|c|c|c|c|}
\hline & \multicolumn{3}{|c|}{$\begin{array}{l}\text { Determinación de susceptibilidad por } \\
\qquad \text { CIM }(\mu \mathrm{g} / \mathrm{mL})\end{array}$} & \multicolumn{3}{|c|}{$\begin{array}{l}\text { Determinación de susceptibilidad por } \\
\text { disco (halo en } \mathrm{mm} \text { ) }\end{array}$} \\
\hline & s & 1 & $\mathbf{R}$ & s & I & $\mathbf{R}$ \\
\hline Staphylococcus aureus & $\leq 2$ & $4-8^{*}$ & $\geq 16$ & No & No & No \\
\hline Staphylococcus coagulasa negativa & $\leq 4$ & $8-16$ & $\geq 32$ & No & No & No \\
\hline Enterococcus spp & $\leq 4$ & $8-16$ & $\geq 32$ & $\geq 17$ & $15-16$ & $\leq 14$ \\
\hline
\end{tabular}


vancomicina. Esta menor respuesta clínica con cepas de ese rango de CIM no ha sido consistentemente encontrada, lo que se analiza más adelante.

Según el modelo farmacocinético/farmacodinámico de antimicrobianos, más conocido por su sigla en inglés PK/ $\mathrm{PD}^{10}$, la actividad antibacteriana de vancomicina se establece como mayoritaria, pero no exclusivamente, concentración dependiente, donde su acción farmacodinámica óptima requiere de un cierto tiempo de una determinada concentración por sobre la CIM para la bacteria, conocido como área bajo la curva ( $\mathrm{ABC} / \mathrm{CIM}$ o, en inglés, $\mathrm{AUC} /$ $\mathrm{MIC}$ ), por lo que corresponde a un mecanismo concentración y tiempo dependiente ${ }^{11}$. Estudios han determinado que el $\mathrm{ABC} / \mathrm{CIM}$ óptimo para reducir el riesgo de fracaso de vancomicina en neumonía y bacteriemia por SARM es $\geq 400^{12,13}$. Debido a la necesidad de lograr un éxito terapéutico minimizando la toxicidad, se ha incorporado la medición de concentraciones plasmáticas (CPs) de vancomicina en las guías clínicas, con el objetivo de alcanzar valores de concentración valle en estado estacionario entre $15-20 \mu \mathrm{g} / \mathrm{mL}$, donde al aplicar la simulación farmacocinética de Montecarlo existe correlación con el $\mathrm{ABC} / \mathrm{CIM}$ deseado. Análisis más completos y complejos en lo farmacodinámico y farmacocinético del fármaco han sido reportados en otras publicaciones ${ }^{14,15}$.

\section{Toxicidad}

Las formulaciones actuales de vancomicina son más purificadas que las originales, lo que se traduce en menor toxicidad. Las toxicidades clásicas han sido renal y ótica, ambas altamente dependientes de la dosis, tiempo de exposición al fármaco, co-medicación nefro y ototóxica, co-morbilidades y edad. También se ha descrito alergia cutánea, neutropenia, eosinofilia y fiebre, en mucha menor frecuencia. Un fenómeno interesante es el denominado síndrome de hombre rojo, caracterizado por exantema eritematoso, prurito y a veces angioedema en la cara, cuello, brazos y tronco superior. El cuadro es de instalación rápida durante la infusión endovenosa del medicamento, y claramente relacionado a la velocidad de infusión, lo que ha llevado a recomendar su administración en $\geq 60 \mathrm{~min}$; este fenómeno parece estar mediado por liberación de histamina por parte de los mastocitos sin mediar un proceso inmunológico ${ }^{16}$.

\section{Agentes etiológicos y las patologías en las cuales se considera el uso de vancomicina}

El uso de vancomicina se dirige fundamentalmente a patologías causadas por cocáceas grampositivas: $S$. aureus, SCN (S. epidermidis y variantes), Enterococcus spp, algunos bacilos grampositivos (en especial C. difficile) y para cepas excepcionales de $S$. pneumoniae resistentes a $\beta$-lactámicos (especialmente en el contexto de meningitis en niños). Frente infecciones por $S$. aureus, su uso se debe reservar para las cepas de SARM que requieran terapia parenteral, ya sea para su variante hospitalaria o la adquirida en la comunidad (SARM-AC). El fármaco es una opción frente a $S$. aureus sensible a meticilina (SASM) en caso de alergia significativa a $\beta$-lactámicos, pero los resultados terapéuticos son inferiores a los obtenidos con $\beta$-lactámicos ${ }^{17}$. SARM es uno de los agentes más frecuentes asociados a IAAS (ver más adelante). También se usa en diarrea por $C$. difficile, con modalidad de uso diferente ${ }^{18}$.

\section{Frecuencia de agentes etiológicos tratables con vancomicina y su perfil de resistencia}

En Chile, el Programa de Control de Infecciones del Ministerio de Salud establece algunos requerimientos de vigilancia de IAAS que implica que los establecimientos públicos tienen la obligación de informar a través de una plataforma digital (SICARS) los agentes etiológicos de las IAAS vigilables. En el resumen del año 2017 -accesible desde la red pública de salud en la página web del sistema $^{19}$ - se constató que prácticamente $2 / 3$ de las cepas de $S$. aureus aisladas tanto de pacientes en hospitalización general como de unidades de pacientes críticos fueron susceptibles a las isoxazolilpenicilinas (cloxacilina, flucloxacilina) en un rango de 39 a 94\%, es decir, correspondían a SASM y no SARM. El GCRB mantiene una vigilancia de resistencia bacteriana que incluye, entre otros, a $S$. aureus, E. faecalis y E. faecium ${ }^{20}$. En esta vigilancia de resistencia, que incluye pacientes de unidades críticas y no críticas, la susceptibilidad encontrada en cocáceas grampositivas se ha mantenido con tendencia a la mejoría en el caso de $S$. aureus respecto a cloxacilina (que en 2009 y 2012 era cercana a 42\%, hasta llegar en 2017 a 62\%) y ligera tendencia al empeoramiento en $E$. faecium respecto a vancomicina. Enterococcus faecalis permanece estable en el tiempo con $99 \%$ de susceptibilidad. La susceptibilidad reportada para adultos se muestra en la Tabla 2.

Un fenómeno que se ha postulado es el del paulatino aumento de la CIM de vancomicina para SARM a nivel

$\begin{aligned} & \text { Tabla 2. Susceptibilidad de cepas de S. aureus, E. faecalis y E. faecium a vancomicina } \\
& \text { reportada en Chile }{ }^{21,22}\end{aligned}$
\begin{tabular}{lccc}
\hline Susceptibilidad por año & $\mathbf{2 0 0 9 ^ { 2 0 }}$ & $\mathbf{2 0 1 2}^{\mathbf{2 1}}$ & $\begin{array}{c}\mathbf{2 0 1 7}^{*} \\
\text { (datos no } \\
\text { publicados) }\end{array}$ \\
\hline S. aureus susceptibles a meticilina (SASM) & $42 \%$ & $42 \%$ & $62 \%$ \\
\hline E. faecalis susceptibles a vancomicina & $99 \%$ & $95 \%$ & $99 \%$ \\
\hline E. faecium susceptibles a vancomicina & $31 \%$ & $24 \%$ & $28 \%$
\end{tabular}


global. Los autores no encontraron datos publicados en el país; pero un meta análisis a nivel global no encontró que el promedio de CIM haya variado en un período de más de 10 años ${ }^{23}$.

\section{Patologías relacionadas a Staphylococcus aureus}

Sin dudas, el patógeno más importante para el uso de vancomicina es $S$. aureus, especialmente en su variedad resistente a meticilina, excluyendo la infección por $C$. difficile. Las patologías más trascendentes causadas por S. aureus y las otras cocáceas grampositivas mencionadas -la inmensa mayoría IAAS- son las siguientes:

\section{Neumonía nosocomial (NN)}

En el país la NN no asociada a ventilación mecánica (VM) es la segunda causa de IAAS y la asociada a VM es la sexta ${ }^{24}$; su etiología es variada pero fundamentalmente bacteriana, predominando bacilos gramnegativos, pero con un rol importante de $S$. aureus, aunque no de otras cocáceas grampositivas $(S$. pneumoniae en caso de NN precoz, es la excepción). Estadísticas internacionales mencionan a $S$. aureus como causa de 14 a $36 \%$ de las no asociadas a VM y en 24 a $32 \%$ en las $\mathrm{NN}$ con $\mathrm{VM}^{25}$ con un porcentaje decreciente en el período más reciente. En Chile, un extenso estudio de NN asociada a VM mostró que $S$. aureus causaba $20 \%$ de los casos (S. pneumoniae en $0,5 \%$ ), siendo más de $70 \%$ de los casos causados por bacilos gramnegativos ${ }^{19}$.

\section{Infecciones del torrente sanguíneo (ITS)}

Pueden ser de adquisición comunitaria o nosocomial y en ambos casos se considera que $S$. aureus es un patógeno relevante, aunque en Chile, ITS de origen comunitario rara vez corresponde a cepas de SARM. En ITS nosocomial, SARM es mucho más frecuente siendo uno de los principales agentes causales. El principal factor de riesgo de ITS nosocomial es el uso de catéteres endovasculares, principalmente catéteres venosos centrales (CVC), en especial los destinados a acceso vascular transitorio para hemodiálisis (aunque frecuentemente de uso prolongado por retardo en implementar un acceso vascular definitivo). El mismo reporte centralizado nacional del sistema SICARS, con datos de 77 hospitales en el año 2017, señalaba que SCN y $S$. aureus eran las cepas más frecuentemente aisladas en ITS asociada a CVC de adultos con $23 \%$ cada una y E. faecalis en $3 \%{ }^{24}$.

\section{Otras IAAS}

Sólo alrededor de $1 \%$ de las infecciones asociadas a catéteres urinarios son causadas por especies de Staphylococcus y $12 \%$ por Enterococcus spp $^{24}$. Para otras IAAS no hay estadísticas nacionales, aunque dada la mayor facilidad de diagnóstico etiológico, la terapia empírica es muchas veces menos urgente y está dirigida por los resultados de los cultivos. En cuanto a infecciones del sitio quirúrgico, alrededor de $50 \%$ de las infecciones con obtención de cultivo muestran la presencia de $S$. aureus.

\section{Patologías relacionadas a otros microorganismos tratables con vancomicina}

En relación a $S$. epidermidis y otros $\mathrm{SCN}$, hay que considerar que estos agentes son parte de la microbiota cutánea normal y su aislamiento siempre debe requerir una evaluación de su rol patógeno real o si son meros colonizantes/contaminantes. Sin lugar a dudas, tienen un rol patógeno frecuente, especialmente en infecciones asociadas a material extraño (catéteres, drenajes, prótesis, material de osteosíntesis, etc.) o en hospederos profundamente inmunodeprimidos, y causan infecciones en los mismos parénquimas que $S$. aureus, excepto pulmón, y en mucho menor grado infecciones de piel y tejidos blandos donde son un frecuente contaminante. Destaca su rol preponderante en ITS asociada a CVC, endocarditis en válvula protésica y otros dispositivos, así como también a artritis séptica en pacientes con prótesis articular. En el tracto urinario, la especie de mayor rol patógeno es $S$. saprophyticus, que rara vez es nosocomial. La microbiota cutánea normal alberga cepas que son, en su mayoría, sensibles a $\beta$-lactámicos. Sin embargo, las cepas infectantes, generalmente a consecuencia de una IAAS, ya han desarrollado mayoritariamente resistencia a estos agentes, independientemente del fenómeno adicional de heterogeneidad de la expresión de resistencia de estas cepas ${ }^{26}$.

\section{Enterococcus spp}

Constituidos principalmente por E. faecalis y E. faecium, están entre los cinco primeros agentes etiológicos de IAAS. Causan patologías comunitarias o nosocomiales. Son agentes de bacteriemia y endocarditis, infecciones del tracto urinario, infecciones intra-abdominales y pélvicas (generalmente post-operatorias) y son frecuente componentes de la infección polimicrobiana del pie diabético. Tradicionalmente, el tratamiento fue con penicilina o ampicilina, asociadas o no a gentamicina pero, debido a un progresivo desarrollo de resistencia a $\beta$-lactámicos, surgió vancomicina como una alternativa. Sin embargo, se ha perdido actividad para la mayoría de las cepas de $E$. faecium (enterococos resistente a vancomicina [ERV]).

\section{Infecciones por Clostridium spp}

Estos microorganismos pueden causar infecciones invasoras o toxigénicas ( $C$. botulinum, C. perfringens), y en ellas, vancomicina es un agente terapéutico de alter- 
Tabla 3. Jerarquización en frecuencia de las etiologías de diversas infecciones asociadas a la atención de salud (24)

$\begin{array}{clll}\text { Ranking } & \text { NAVM } & \text { ISQ* } & \text { ITS/CVC** } \\ 1^{\circ} & \text { P. aeruginosa (25\%) } & \text { K. pneumoniae (24\%) } & \text { S. aureus (17\%) } \\ 2^{\circ} & \text { S. aureus (22\%) } & \text { S. aureus (24\%) } & \text { K. pneumoniae }(16 \%) \\ 3^{\circ} & \text { K. pneumoniae }(21 \%) & \text { Staphylococcus coag (-) (12\%) } & \text { S. epidermidis (15\%) } \\ 4^{\circ} & \text { Acinetobacter spp (12\%) } & \text { S. epidermidis (12\%) } & \text { P. aeruginosa (13\%) }\end{array}$

Último acceso septiembre 2018 para data 2017. *de 36 infecciones 17 se identificó agente. NAVM: neumonía asociada a ventilación mecánica. ISQ: infección de sitio quirúrgico (aquí*: cirugía coronaria). **ITS/CVC: infección del torrente sanguíneo asociado a catéter venoso central. ITU/CUP: infección del tracto urinario asociado a catéter urinario permanente. ${ }^{* *}$ ITU/CUP en UPC.

nativa si hay alergia a $\beta$-lactámicos. Sin embargo, para la infección por $C$. difficile, mayoritariamente IAAS, y de alta incidencia nacional y mundial, vancomicina es recomendada actualmente como antimicrobiano de primera elección ${ }^{18}$. Esta patología es la única donde vancomicina debe usarse vía oral o en enema; téngase presente que la administración parenteral es inefectiva (pero es esta formulación galénica la indicada para administración por vía oral o rectal).

La Tabla 3 jerarquiza por rango de frecuencia los principales agentes de los agentes etiológicos de las patologías mencionadas según datos de SICARS.

\section{Consideraciones terapéuticas generales}

Vancomicina es un antimicrobiano de potencia moderada y de un estrecho margen terapéutico, que requiere CPs muy superiores a la CIM para los patógenos causales de la infección a tratar, especialmente contra $S$. aureus $\mathrm{y}$ en pacientes bacteriémicos. Tiene una farmacocinética compleja y una llegada escasa a muchos parénquimas tales como el ojo, cerebro y meninges, o variable como en pulmón y hueso, pero alta (similar a plasma o moderadamente inferior) en el riñón, hígado, miocardio, tejidos blandos y paredes vasculares. En los parénquimas de escasa llegada, rara vez se logran concentraciones terapéuticas con las dosis habituales o sin administración local (i.e. intratecal). $\mathrm{Su}$ excreción es primariamente renal, como producto activo, por lo que se acumula en presencia de insuficiencia renal; no es hemodializable pero se puede llegar a eliminar en cantidades no menores con los actuales procedimientos de diálisis que utilizan membranas de alto flujo con mayor permeabilidad, o durante hemofiltración.

Su dosificación habitual es por peso, tanto la dosis de carga, 20-30 mg/kg, como de mantención, $15 \mathrm{mg} / \mathrm{kg}$ por dosis, con un máximo de $2 \mathrm{~g}$ por dosis y $4 \mathrm{~g}$ diarios, pero dependiendo del patógeno y sitio de infección, esta dosis puede requerir incrementos importantes para alcanzar concentraciones durables adecuadas. Inicialmente se administraba cada $6 \mathrm{~h}$, luego se popularizó la dosis cada 12 h que es la forma estándar actual, aunque en muchos casos se debe incrementar la dosis y/o frecuencia frente a patógenos de CIM alta, parénquimas de llegada restringida y por la no obtención de concentraciones adecuadas. Si bien en cuadros de satisfactoria respuesta puede darse la dosis de mantención desde un inicio (ej. infecciones de tejidos blandos), para otras infecciones por S. aureus (bacteriemias y en infecciones invasoras de otros parénquimas), se recomienda la obtención rápida de concentraciones terapéuticas con dosis de carga (máximo 2 gramos, o 3 gramos en obesos). Aunque esto ayuda a obtener la meta farmacocinética ${ }^{15,27}$, no hay consenso en que esto logre un beneficio clínico ${ }^{28}$. Las dosis a usar basados en modelos simples y habituales de la función renal para obtener las CPs deseadas son poco precisas dada la gran variabilidad inter e intrapersonal de la farmacocinética del antimicrobiano, pero el Modelo de Montecarlo, algo más complejo, mejora la predicción ${ }^{29}$. La medición de CPs es fácil, rápida y de bajo costo; la meta a obtener para las infecciones graves ya mencionadas es CPs valle (pre dosis) entre 15-20 $\mu \mathrm{g} / \mathrm{mL}$ inmediatamente antes de la cuarta dosis o $25-30 \mu \mathrm{g} / \mathrm{mL}$ en caso de infusión continua, y se ha usado en la práctica como sustituto de la medición más compleja de ABC/CIM, que, además, puede tener importantes variaciones según cómo se determine la CIM. Recientemente se ha revitalizado el cálculo de ABC/CIM con fuerte recomendación de sustituir el monitoreo del tratamiento con vancomicina, desde el parámetro exclusivo de CPs a la meta ABC/CIM (con la determinación de dos concentraciones), donde el cálculo optimizado por modelo bayesiano puede ser realizado en algoritmos obtenibles a través de aplicaciones en dispositivos electrónicos ${ }^{30}$.

A pesar de la fácil obtención de CPs, el medicamento es frecuentemente administrado en forma inapropiada ${ }^{31,32}$. Debe tenerse presente que la frecuencia de nefrotoxicidad con concentraciones $\geq 15 \mu \mathrm{g} / \mathrm{mL}$ es mayor, por lo que la función renal debe monitorearse con particular dedicación, especialmente si se asocia a otras medicamentos con potencial nefrotóxico, destacando la sinergia nefro- 
tóxica de la asociación vancomicina con piperacilina/ tazobactam $^{33}$.

Con las dosis estándares de $30 \mathrm{mg} / \mathrm{kg} /$ día $(1 \mathrm{~g}$ cada $12 \mathrm{~h}$ en promedio) un porcentaje importante de los pacientes con función renal normal no alcanzan las CPs deseadas ni un valor AUC/CIM de 400, requiriendo mayores dosis que el estándar tradicional, especialmente en pacientes jóvenes o estados hiperdinámicos (i.e quemados). La dosis en obesos debe ser determinada por peso ajustado; al igual que en pacientes con disfunción renal, la determinación de CP valle adquiere especial importancia ${ }^{15}$.

En pacientes con insuficiencia renal se asiste a varios escenarios:

- Paciente con disfunción estable en quienes la dosis de mantención debe ser más baja (no así la inicial) y ajustada ya sea por cálculo predictor o por CP;

- Paciente con función renal cambiante (deterioro o recuperación) en quien los ajustes pueden ir desfasados con la realidad del momento; en ellos el monitoreo con CPs es esencial, aunque el momento de determinar la concentración valle puede ser más complejo y a veces se debe recurrir a determinación de CPs fuera ese plazo, pero alejadas de la última dosis; en ese caso, debe estar en el rango deseado según el patógeno y el sitio de infección, pero siempre $\leq 20 \mu \mathrm{g} / \mathrm{mL}$.

- Pacientes con terapia de reemplazo renal (hemodiálisis, peritoneodiálisis o diálisis continua). La dosis de carga es independiente de la función renal, por lo que no debe ser modificada, así como las concentraciones a alcanzar según la patología y el patógeno. Para las siguientes dosis hay distintas estrategias según el tipo de diálisis donde el mejor método de administración permanece incierto. Las recomendaciones con la hemodiálisis intermitente consisten en medir CPs pre diálisis y según ellos, guiar la dosis que corresponda post diálisis, asumiendo que los filtros modernos pueden llegar a retirar entre $30-40 \%$ de la vancomicina plasmática en 3 a 5 h. Sólo hay que dejar dosis fijas en pacientes en quienes las concentraciones han sido adecuadas y estables, dando dosis mayor a pacientes con función renal residual comparada con pacientes anúricos. Es importante destacar que la obtención de muestra para medir CPs post hemodiálisis (si es que se desea tomar pre y post), deben ser tomados entre 4 y $6 \mathrm{~h}$ posteriores al fin de la hemodiálisis, ya que se genera un fenómeno de redistribución plasmática que puede llevar a que la concentración aparente sea menor a lo real. En pacientes bajo hemofiltración veno-venosa continua, método capaz de remover vancomicina más eficientemente, se sugiere una dosis diaria de 7,5-10 $\mathrm{mg} / \mathrm{kg}$ y ajustar con CP. En el caso de pacientes en peritoneodiálisis, una dosis única intraperitoneal de 30 $\mathrm{mg} / \mathrm{kg}$ semanal o menores, cada cinco días, puede puede alcanzar CP óptimas para tratar infecciones peritoneales asociadas al catéter ${ }^{34,35}$.
Hay experiencia con administración continua (24 h), con el objeto de disminuir la nefrotoxicidad o aumentar su efectividad; lo reportado es variable en ambos objetivos, pero podrían existir ventajas en algunas subpoblaciones como pacientes en hemofiltración veno-venosa continua y paciente crítico neuroquirúrgico ${ }^{37}$.

\section{Paciente crítico}

En el paciente crítico con disfunción de órganos varios, contenido acuoso con volumen de distribución y hemodinamia variable, la farmacocinética de vancomicina es particularmente poco predecible y los modelos predictores habituales son menos precisos. Modelos que incorporan las denominadas variables PK fisiopatológicas han permitido alcanzar y mantener con mayor precisión CPs adecuadas ${ }^{29}$

\section{Efectividad terapéutica}

Vancomicina no es un antimicrobiano de primera selección, excepto para $C$. difficile, y es de alternativa frente a cocáceas grampositivas resistentes o por alergia a $\beta$-lactámicos. Como fármaco de alternativa, su efectividad comparativa frente a patógenos no resistentes a antimicrobianos de primera línea es, en prácticamente todas las localizaciones, inferior ${ }^{17}$ y no debiera usarse, a menos que existan esas situaciones ya definidas. Esta menor eficacia se ha demostrado claramente frente a uso de vancomicina en pacientes con infección por SASM que reportan alergia a $\beta$-lactámicos o en hemodiálisis por comodidad posológica ${ }^{38,39}$.

El problema fundamental radica en su uso empírico en aquellas condiciones donde las probabilidades ya sea de infección bacteriana, infección bacteriana estafilocócica o infección estafilocócica resistente a meticilina, son bajas. Aquí se arriesga a someter a personas innecesariamente a antibioterapia o, de ser necesarias, a que no sea la terapia óptima para la condición. Obviamente, en muchas situaciones el uso empírico solo o asociado es apropiado y necesario. Diferenciar adecuadamente ambas situaciones requiere de buen conocimiento epidemiológico local, buen juicio clínico y adecuadas herramientas diagnósticas. Dado que no es un antimicrobiano óptimo, vale la pena analizar los resultados terapéuticos en situaciones donde sí está indicada de primera línea, básicamente en infecciones por SARM. Un aspecto importante respecto a la dosificación se refiere a la efectividad según la CIM para el patógeno, específicamente para $S$. aureus. Con cepas con $\mathrm{CIM}>1,5 \mu \mathrm{g} / \mathrm{mL}(\mathrm{o} \geq 2 \mu \mathrm{g} / \mathrm{mL})$ el valor óptimo de $\mathrm{ABC} /$ CIM se obtiene en una minoría de casos y se ha reportado mayor fracaso terapéutico, lo que ha llevado a recomendar alternativas a vancomicina en estas situaciones ${ }^{7}$. Sin embargo, esta asociación no ha sido ratificada en forma 
incontrarrestable y hay meta-análisis y revisiones sistemáticas recientes que no lo respaldan y que sostienen que el sitio de infección es más determinante ${ }^{40,41}$. La posición de la Infectious Disease Society of America de E.U.A., es que, dada la variabilidad de resultado de CIM según el método a usar, especialmente al nivel de 1 a $2 \mu \mathrm{g} / \mathrm{mL}$, con cepas de hasta $2 \mu \mathrm{g} / \mathrm{mL}$ se evalúe la eficacia terapéutica clínica y microbiológica de vancomicina más que recurrir obligatoriamente a una selección de alternativa ${ }^{42}$. Estas consideraciones no han sido estudiadas en profundidad contra otras especies.

\section{Neumonía nosocomial}

De los agentes causales de NN, sólo $S$. aureus es relevante para el uso de vancomicina. Las alternativas terapéuticas a vancomicina se reducen, dada la menor eficacia de tigeciclina (no aprobada para esta indicación) así como la inactivación pulmonar de daptomicina. El principal competidor ha sido linezolid, con estudios de eficacia terapéutica comparada variable. Una revisión sistemática y meta-análisis no encontró diferencias significativas ${ }^{43}$. Ceftarolina, cefalosporina con acción anti SARM ha mostrado resultados preliminares prometedores en NN estafilocócica ${ }^{44}$.

\section{Sepsis por catéter y bacteriemia}

La efectividad terapéutica de vancomicina en el tratamiento de infecciones por SARM es menor al de cuadros similares por SASM tratados con $\beta$-lactámicos anti estafilocócicos. A pesar de ello, vancomicina se mantiene aún como el estándar terapéutico en esta condición, pero para toda bacteriemia por SARM, en especial si la CIM es $>1 \mu \mathrm{g} / \mathrm{mL}$ se debe intentar conseguir CPs valle de vancomicina entre 15 y $20 \mu \mathrm{g} / \mathrm{mL}$ y evaluar la respuesta clínica. Se ha planteado una eventual mejor respuesta de daptomicina, especialmente en casos de CIM alta en rango de sensibilidad microbiológica de vancomicina ${ }^{45} \mathrm{y}$ es el antibacteriano de elección en casos de bacteriemias por SARM con CIM $>2 \mu \mathrm{g} / \mathrm{mL}$. Otras alternativas son menos competitivas, ya sea por no ser indicación habitual en bacteriemia (linezolid y tigeciclina), o ser inferiores (cotrimoxazol) ${ }^{46}$.

En las infecciones por SCN, entre las que destacan las bacteriemias, se enfrenta el doble problema de diferenciar, tanto la colonización/contaminación del eventual rol patógeno del agente, así como la caracterización del antibiotipo entre cepas sensibles a meticilina, de las resistentes, ya que muchas veces las poblaciones bacterianas son heterogéneas y expresan variablemente su fenotipo de resistencia, lo que otorga más incertidumbre a la actividad de un $\beta$-lactámico anti-estafilocócico. Para las cepas de SCN se exige una CIM más baja de cloxacilina para catalogarlos como sensibles $(\leq 0,25 \mu \mathrm{g} / \mathrm{mL})$ que para $S$. aureus. Por estas razones, vancomicina es frecuentemente utilizada de partida dada su estable y confiable actividad (excepto para S. haemolyticus y lugdunensis).

\section{Endocarditis infecciosa (EI)}

SASM, SARM, SCN y Enterococcus spp son agentes habituales de endocarditis, tanto en válvula natural como protésica. Para EI por SARM, SCN y Enterococcus spp, vancomicina juega un rol importante. Dada la rareza de EI por SCN en válvula nativa, las recomendaciones de manejo para EI prácticamente no se pronuncian específicamente sobre esta condición y se debe asumir el mismo tratamiento que por SARM (a menos que la susceptibilidad a cloxacilina sea concluyente). En cambio para toda EI estafilocócica por S. aureus o SCN resistente a meticilina, con material intracardíaco protésico, se recomienda terapia combinada con rifampicina y gentamicina adicional a vancomicina ${ }^{42}$.

\section{Ventriculitis y meningitis}

Los cuadros que requieren uso de vancomicina son generalmente IAAS post operatorias neuro-quirúrgicas, muchas de ellas asociadas a la colocación de dispositivos de drenaje descompresivo de líquido cefalorraquídeo. Staphylococcus aureus -en proporción variable SARMy SCN son, junto a bacilos gramnegativos, los agentes causales más frecuentes de estas complicaciones y vancomicina forma parte del enfoque terapéutico empírico inicial. Las meninges son relativamente impermeables a vancomicina, aunque su penetración mejora en caso de inflamación, y, a su vez, se reduce por efecto del uso de corticosteroides, recomendados en meningitis neumocócica y por $H$. influenzae, de ahí que se deba utilizar dosis máxima, incluso por vía intratecal (especialmente en ventriculitis). Hay autores que recomiendan, por un lado, la asociación con rifampicina y, por otro, que el umbral para considerar terapia alternativa a vancomicina sea una CIM de ésta de $\geq 1 \mu \mathrm{g} / \mathrm{mL}^{47}$.

\section{Otras infecciones}

En infecciones por SARM de piel y tejidos blandos vancomicina es de primera línea si se requiere terapia parenteral, pero en casos susceptibles de terapia oral, buenas alternativas son cotrimoxazol, tetraciclinas y clindamicina, en especial frente a SARM-AC. En osteomielitis y artritis por SARM, vancomicina está indicada, con rifampicina asociada en la fase inicial en presencia de material extraño o protésico (por su efecto beneficioso sobre las biopelículas), con reemplazo a largo plazo posterior por combinaciones de antimicrobianos orales activos.

\section{Infección por $C$. difficile}

No es el propósito de esta presentación analizar en detalle esta indicación, excepto para mencionar que vancomicina oral es la terapia de elección, pero sin actividad por vía parenteral ${ }^{18}$. 


\section{Uso profiláctico de vancomicina}

La profilaxis antimicrobiana pre-operatoria más ampliamente usada es con cefazolina al inicio del procedimiento; con extensión excepcional por hasta $24 \mathrm{~h}$ post-operatorias, sin evidencias de beneficio con la subsecuente administración. Dada la aparición de infecciones post-operatorias causadas por SARM y SCN en pacientes con cirugía cardíaca, vascular, traumatológica y neuro-quirúrgica, en especial aquellas con colocación de cuerpos extraños o material protésico, se ha considerado el uso de vancomicina profiláctica en algunas de estas situaciones. Esto no debiera ser rutinario, dada su inferior efectividad general. Sólo debería considerarse cuando la tasa de infecciones por SARM alcanza un nivel superior a un estándar prefijado o en pacientes conocidamente colonizados por SARM. Debe tenerse presente que, dada la necesidad de una lenta administración de vancomicina, rara vez se puede iniciar en el pabellón quirúrgico como está indicado en las otras profilaxis y debe coordinarse adecuadamente su administración con la sala de hospitalización pre-operatoria del paciente cuando, por razones prácticas, no se inicie en pabellón.

\section{Errores frecuentes en el uso de vancomicina}

Vancomicina es frecuentemente usada en forma inadecuada, ya sea en indicación, dosificación inicial y de mantención, como en monitoreo. Con base a la acumulación de años de estudios locales de prevalencia de uso de antimicrobianos como parte de los programas de IAAS, y también como la experiencia de los autores en calidad de médicos clínicos en infectología de adultos, microbiología, y farmacia clínica, los autores han identificado como errores frecuentes a tres categorías que se describen en la Tabla 4.

\section{Conclusiones}

Vancomicina es un antimicrobiano sub-óptimo de acuerdo a criterios contemporáneos: antiguo, de potencia moderada, con bajo margen terapéutico, con perfil de seguridad discreto y farmacocinética variable, que obliga al monitoreo de CPs en casos graves o de concentraciones inciertas.

Se asiste a una creciente disminución de sensibilidad de algunos de los patógenos para los que está indicada ${ }^{48,49}$.

Se sobre-utiliza por exagerada sospecha de infección frente a aparición de cuadros febriles, compromiso respiratorio o hemodinámico, entre otros, que frecuentemente tienen etiología no infecciosa, viral o son auto-limitados.

Hay una sobre-estimación, tanto de la presencia de cocáceas grampositivas resistentes a $\beta$-lactámicos en cuadros genuinamente infecciosos bacterianos, como de una verdadera alergia a fármacos de primera línea más eficaces.

Hay, por lo tanto, frecuentes errores en la indicación, dosificación y forma de administración. Sin embargo, la

\begin{tabular}{|c|c|}
\hline Categorías de errores & Descripción de errores \\
\hline Indicación & $\begin{array}{l}\text { - No hay una enfermedad infecciosa } \\
\text { - Microorganismo etiológico susceptible a otro antibacteriano de primera línea } \\
\text { - Se documenta infección por microorganismo resistente a vancomicina (i.e. bacilo gramnegativo) } \\
\text { - Inicio sin toma de exámenes adecuados } \\
\text { - Asociación con otro antibacteriano en ausencia de sinergia validada } \\
\text { - Asociación con otro antibacteriano o fármaco con toxicidad compartida } \\
\text { - Uso de vancomicina por supuesta alergia a } \beta \text {-lactámico que no se ha documentado }\end{array}$ \\
\hline Dosificación & $\begin{array}{l}\text { - No se da la dosis de carga recomendada en infecciones graves o por SARM } \\
\text { - Uso de dosis fija estándar (sin ajuste por peso) } \\
\text { - Rápida velocidad de administración } \\
\text { - Ausencia de ajuste dinámico por función renal }\end{array}$ \\
\hline Interpretación & $\begin{array}{l}\text { - Inadecuada interpretación microbiológica de los resultados de (i.e. cultivos contaminantes o microbiota no } \\
\text { infectante, punta de catéter positivo sin bacteriemia o sepsis) }\end{array}$ \\
\hline Monitoreo & $\begin{array}{l}\text { - No documentación de esterilización de la sangre frente a bacteriemia por SARM. } \\
\text { - Ausencia de control con CPS } \\
\text { - Control de CPs en momento inapropiado } \\
\text { - Ausencia de ajuste de acuerdo a CPs a pesar de contar con resultado confiable }\end{array}$ \\
\hline
\end{tabular}


alta prevalencia de patógenos (sospechados o documentados), que se han hecho resistentes a antimicrobianos de primera línea y el bajo costo de vancomicina han mantenido su vigencia.

Los antimicrobianos alternativos, antiguos y de reciente desarrollo, muchas de ellos de costo considerablemente superior, no han implicado una sustancial mejoría terapéutica que lleve al desuso generalizado del producto.

Una adecuada comprensión de las fortalezas y debilidades del antimicrobiano, del cambiante panorama epidemiológico de patologías y de los agentes infecciosos para los que se usa, asociado a prácticas adecuadas de administración y monitoreo, permitirán sacar el mejor beneficio terapéutico de esta necesaria y aún vigente herramienta terapéutica.

Esta revisión, enfocada primariamente en medicina de adultos, ha tenido como objetivo cumplir con ese propósito.

\section{Resumen}

Introducción: Vancomicina, terapia estándar para enterococos y estafilococos resistentes a $\beta$-lactámicos tradicionales (Staphylococcus aureus [SARM] y Sta- phylococcus coagulasa negativa), tiene extenso uso empírico en infecciones nosocomiales. Farmacológicamente débil, de estrecho margen terapéutico y farmacocinética poco predecible, es un fármaco sub-estándar según criterios contemporáneos. Tiene excesivo uso, por sobrediagnóstico de infecciones bacterianas y, en infecciones genuinas, por sobre-estimación etiológica de patógenos $\beta$-lactámico-resistentes. Últimamente han surgido nuevas amenazas a su efectividad: peores desenlaces en infecciones por SARM con CIM en rango alto de sensibilidad y resistencia de enterococos. Hay frecuente administración inadecuada en: dosis e intervalos, ausencia de dosis de carga inicial, falta de monitoreo con concentraciones plasmáticas, inadecuada dosificación en presencia de insuficiencia renal o diálisis e, importantemente, mantención de uso en ausencia de clara documentación de su necesidad. Nuevos fármacos anti-estafilocócicos no han permitido un reemplazo generalizado de vancomicina por lo que ésta mantiene un importante rol en la medicina contemporánea. Conclusiones: Una comprensión de las fortalezas y debilidades del fármaco, así como de la cambiante epidemiología y propiedades microbiológicas de los patógenos relevantes, al igual que un uso prudente y selectivo, permitirán optimizar su uso y mantener su rol terapéutico en la medicina actual y futura.

\section{Referencias bibliográficas}

1.- Magill S S, Edwards J R, Beldavs Z G, Dumyati G, Janelle S J, Kainer M A, et al. Prevalence of antimicrobial use in US acute care hospitals, May-September 2011. JAMA 2014; 312 (14): 1438-46. doi: 10.1001/ jama.2014.12923.

2.- Domínguez I, Rosales R, Cabello Á, Bavestrello L, Labarca J. Evaluación del consumo de antimicrobianos en 15 hospitales chilenos: Resultados de un trabajo colaborativo, 2013. Rev Chilena Infectol 2016; 33 (3): $307-$ 12. doi: 10.1001/jama.2014.12923.

3.- Deresinski S. Counterpoint: Vancomycin and Staphylococcus aureus-an antibiotic enters obsolescence. Clin Infect Dis 2007; 44 (12): 1543-8. doi:10.1086/518452.

4.- Waters C D, Caraccio J. Rate of positive cultures necessitating definitive treatment in patients receiving empiric vancomycin therapy. Infect Control Hosp Epidemiol 2018; 39 (8): 989-90. doi: 10.1017/ice.2018.123.

5.- Boyce J M, Pop O-F, Abreu-Lanfranco O, Hung W Y, Fisher A, Karjoo A, et al. A trial of discontinuation of empiric vancomycin therapy in patients with suspected methicillin-resistant Staphylococcus aureus health care-associated pneumonia. Antimicrob Agents Chemother 2013; 57 (3): 1163-8. doi: 10.1128/AAC.0196512.
6.- Murray B E, Arias C A, Nannini E

C. Glycopeptides (Vancomycin and Teicoplanin), Streptogramins (QuinupristinDalfopristin), Lipopeptides, Daptomycin), and Lipoglycopeptides (Telavancin) in Mandell, Douglas, and Bennett's Principles and Practice of Infectious Diseases. John E. Bennet, Raphael Dolin and Martin J. Blaser, editores.8a edición, Elsevier 2016.

7.- CLSI. Performance Standards for Antimicrobial Susceptibility Testing. 28th Edition. CLSI Supplement M 100 Wayne, PA. Clinical and Laboratory Standards Institute; 2018.

8.- EUCAST: Clinical breakpoints and dosing of antibiotics [Internet]. [acceso, 16 de enero de 2019]. Disponible en: http://www.eucast.org/ clinical_breakpoints/

9.- Soriano A, Marco F, Martínez J A, Pisos E, Almela M, Dimova VP, et al. Influence of vancomycin minimum inhibitory concentration on the treatment of methicillin-resistant Staphylococcus aureus bacteremia. Clin Infect Dis 2008; 46 (2): 193-200. doi: $10.1086 / 524667$.

10.- Roberts J A, Lipman J. Antibacterial dosing in intensive care: pharmacokinetics, degree of disease and pharmacodynamics of sepsis. Clin Pharmacokinet. 2006; 45 (8): 755-73. doi: 10.2165/00003088-200645080-00001

11.- Varghese J M, Roberts J A, Lipman J.
Antimicrobial pharmacokinetic and pharmacodynamic issues in the critically ill with severe sepsis and septic shock. Crit Care Clin 2011; 27 (1): 19-34. doi: 10.1016/j. ccc.2010.09.006.

12.- Song K H, Kim H B, Kim H S, Lee MJ, Jung Y, Kim G, et al. Impact of area under the concentration-time curve to minimum inhibitory concentration ratio on vancomycin treatment outcomes in methicillin-resistant Staphylococcus aureus bacteraemia. Int $\mathrm{J}$ Antimicrob Agents 2015; 46 (6): 689-95. doi: 10.1016/j.ijantimicag.2015.09.010.

13.- Rybak M, Lomaestro B, Rotschafer JC, Moellering R Jr, Craig W, Billeter M, et al. Therapeutic monitoring of vancomycin in adult patients: a consensus review of the American Society of Health-System Pharmacists, the Infectious Diseases Society of America, and the Society of Infectious Diseases Pharmacists. Am J Health-Syst Pharm 2009; 66 (1): 82-98. doi: 10.2146/ajhp080434.

14.- Mogle B T, Steele J M, Seabury R W, Dang U J, Kufel W D. Implementation of a two-point pharmacokinetic AUC-based vancomycin therapeutic drug monitoring approach in patients with methicillinresistant Staphylococcus aureus bacteremia. Int J Antimicrob Agents Int J Antimicrob Agents 2018; 52 (6): 805-10. doi: 10.1016/j. ijantimicag.2018.08.024. 
15.- Álvarez R, López L E, Molina J, Cisneros J M, Pachón J. Optimizing the clinical use of vancomycin. Antimicrob Agents Chemother. 2016; 60: 2601-9. doi: 10.1128/ AAC.03147-14.

16.- Polk R E, Healy D P, Schwartz L B, Rock D T, Garson M L, Roller K. Vancomycin and the red-man syndrome: pharmacodynamics of histamine release. J Infect Dis. 1988; 157(3): 502-7. doi:10.1093/infdis/157.3.502

17.- McDanel J S, Perencevich E N, Diekema D J, Herwaldt L A, Smith T C, Chrischilles E A, et al. Comparative effectiveness of beta-lactams versus vancomycin for treatment of methicillinsusceptible Staphylococcus aureus bloodstream infections among 122 hospitals. Clin Infect Dis. 2015; 61(3): 361-7. doi: 10.1093/cid/civ308

18.- McDonald L C, Gerding D N, Johnson S, Bakken J S, Carroll K C, Coffin S E, et al. Clinical practice guidelines for Clostridium difficile infection in adults and children: 2017 Update by the Infectious Diseases Society of America (IDSA) and Society for Healthcare Epidemiology of America (SHEA). Clin Infect Dis 2018; 66 (7): 987-94. doi: 10.1093/cid/ cix 1085 .

19.- Infecciones Intrahospitalarias [Internet]. Ministerio de Salud - Gobierno de Chile. [acceso, 16 de enero de 2019]. Disponible en: https://www.minsal.cl/infecciones_ intrahospitalarias/

20.- Labarca L J. Desde el aporte local al control de la resistencia bacteriana en Chile. Rev Chil Infectol 2011; 28 (1): 12-3. doi: $10.4067 /$ S0716-10182011000100002

21.- Silva O F, Cifuentes D M, Pinto C M E. Resultados de la vigilancia de susceptibilidad antimicrobiana en Chile: Consolidando una red. Rev Chilena Infectol 2011; 28 (1): 19-27. doi: 10.4067/S0716-10182014000200002.

22.- Cifuentes D M, Silva F, García P, Bello H, Briceño I, Calvo-A M, et al. Susceptibilidad antimicrobiana en Chile 2012. Rev Chil Infectol. 2014; 31 (2): 123-30. doi: 10.4067/ S0716-10182014000200002.

23.- Díaz R, Ramalheira E, Afreixo V, Gago B. Evaluation of vancomycin MIC creep in Staphylococcus aureus. Clin Microbiol Infect 2018; 24: 97-104. doi.org/10.1016/j. jgar.2017.04.007

24.- Sistema de Indicadores de Calidad y Atención y Resultados Sanitarios (SICARS) en http://www. sicars.minsal.cl. (acceso, 22 de diciembre de 2018).

25.- Jones R N. Microbial etiologies of hospitalacquired bacterial pneumonia and ventilatorassociated bacterial pneumonia. Clin Infect Dis 2010; 51 (Supplement_1): S81-S87. doi: 10.1086/65305.

26.- Pedroso S H S P, Sandes S H C, Filho R A T, Nunes A C, Serufo J C, Farias L M, et al. Coagulase-negative Staphylococci isolated from human bloodstream infections showed multidrug resistance profile. Microb Drug Resist Larchmt N 2018; 24 (5): 635-47. doi: $10.1089 / \mathrm{mdr} .2017 .0309$.

27.- Rosini J M, Laughner J, Levine B J, Papas M A, Reinhardt J F, Jasani $\mathrm{N}$ B. A randomized trial of loading vancomycin in the emergency department Ann Pharmacother 2015; 49: 6-13; doi: $10.1177 / 1060028014556813$.

28.- Reardon J, Lau T T, Ensom M H. Vancomycin loading doses: a systematic review. Ann Pharmacother 2015; 49 (5): 557-65. doi: $10.1177 / 1060028015571163$.

29.- Roberts J A, Kirkpatrick C M J, Lipman J. Monte Carlo simulations: maximizing antibiotic pharmacokinetic data to optimize clinical practice for critically ill patients. J Antimicrob Chemother. 2011; 66(2): 227-31. doi:10.1093/ $\mathrm{jac} / \mathrm{dkq} 449$.

30.- Turner R B, Kojiro K, Shephard E A, Won R, Chang E, Chan D, et al. Review and validation of Bayesian dose-optimizing software and equations for calculation of vancomycin area under the curve in critically ill patients. Pharmacotherapy 2018; 38 (12): 1174-83. doi: 10.1002/phar.2191

31.- Bakke V, Sporsem H, Von der Lippe E, Nordøy I, Lao Y, Nyrerød HC, et al. Vancomycin levels are frequently subtherapeutic in critically ill patients: a prospective observational study. Acta Anaesthesiol Scand 2017 Jul; 61(6): 627-35. doi: 10.1111/aas.12897.

32.- Rodman D P, McKnight J T, Rogers T, Robbins $M$. The appropriateness of initial vancomycin dosing. J Fam Pract 1994; 38: 473-7. PMID: 8176345.

33.- Balcı C, Uzun Ö, Arıcı M, Hayran SA, Yüce D, Ünal S. Nephrotoxicity of piperacillin/ tazobactam combined with vancomycin: should it be a concern? Int J Antimicrob Agents. 2018; 52(2):180-4. doi: 10.1016/j. ijantimicag.2018.03.024

34.- Matsumoto K, Takesue Y, Ohmagari N, Mochizuki T, Mikamo H, Seki M, et al. Practice guidelines for therapeutic drug monitoring of vancomycin: a consensus review of the Japanese Society of Chemotherapy and the Japanese Society of Therapeutic Drug Monitoring. J Infect Chemother 2013; 19 (3): 365-80. doi: 10.1007/s10156-013-0599-4.

35.- Crew P, Heintz S J, Heintz B H. Vancomycin dosing and monitoring for patients with end-stage renal disease receiving intermittent hemodialysis. Am J Health Syst Pharm 2015; 72 (21): 1856-64. doi: 10.2146/ajhp150051.

36.- Zhu L, Zhou Q. Optimal infusion rate in antimicrobial therapy: explosion of evidence in the last five years. Infect Drug Resist. 2018; 11: 1105-7. doi: 10.2147/IDR.S167616

37.- Blumenthal K G, Parker R A, Shenoy E S, Walensky R P. Improving clinical outcomes in patients with methicillin-sensitive Staphylococcus aureus bacteremia and reported penicillin allergy. Clin Infect Dis 2015; 61 (5): 741 doi: 10.1093/cid/civ394.

38.- Stryjewski M E, Szczech L A, Benjamin D K, Inrig J K, Kanafani Z A, Engemann $\mathrm{J} J$, et al. Use of vancomycin or firstgeneration cephalosporins for the treatment of hemodialysis-dependent patients with methicillin-susceptible Staphylococcus aureus bacteremia. Clin Infect Dis 2007; 44 (2): 190-6. doi:10.1086/510386.

39.- Adani S, Bhowmick T, Weinstein M P Narayanan N. Impact of vancomycin MIC on clinical outcomes of patients with methicillinresistant Staphylococcus aureus bacteremia treated with vancomycin at an institution with suppressed MIC reporting. Antimicrob Agents Chemother 2018; 62 (4). pii: e02512-17. doi: 10.1128/AAC.02512-17.

40.- Kalil A C, Van Schooneveld T C, Fey P D, Rupp M E. Association between vancomycin minimum inhibitory concentration and mortality among patients with Staphylococcus aureus bloodstream infections: a systematic review and meta-analysis. JAMA 2014; 312 (15): 1552-64. doi: 10.1097/ CCM.0b013e3181eb3b96.

41.- Liu C, Bayer A, Cosgrove S E, Daum R S, Fridkin S K, Gorwitz R J, et al. Clinical practice guidelines by the Infectious Diseases Society of America for the treatment of methicillin-resistant Staphylococcus aureus infections in adults and children. Clin Infect Dis 2011; 52 (3): e18-55. doi:10.1093/cid/ciq146.

42.- Wang Y, Zou Y, Xie J, Wang T, Zheng X, He $\mathrm{H}$,et al. Linezolid versus vancomycin for the treatment of suspected methicillin-resistant Staphylococcus aureus nosocomial pneumonia: a systematic review employing meta-analysis. Eur J Clin Pharmacol 2015; 71 (1): 107-15. doi: 10.1007/s00228-014-1775-x.

43.- Sotgiu G, Aliberti S, Gramegna A, Di Pasquale $\mathrm{M}$, Trogu F, et al. Efficacy and effectiveness of ceftaroline fosamil in patients with pneumonia: a systematic review and meta-analysis. Respir Res. 2018 Oct 23; 19(1): 205. doi: 10.1186/ s12931-018-0905-x.

44.- Claeys K C, Zasowski E J, Casapao A M, Lagnf A M, Nagel J L, Nguyen C T, et al. Daptomycin improves outcomes regardless of vancomycin mic in a propensity-matched analysis of methicillin-resistant Staphylococcus aureus bloodstream infections. Antimicrob Agents Chemother 2016; 60 (10): 5841-8. doi: 10.1128/AAC.00227-16

45.- Paul M, Bishara J, Yahav D, Goldberg E, Neuberger A, Ghanem-Zoubi N, et al. Trimethoprim-sulfamethoxazole versus vancomycin for severe infections caused by meticillin resistant Staphylococcus aureus: 


\section{Antimicrobianos}

randomised controlled trial. BMJ 2015; 350:

h2219. doi:10.1136/bmj.h2219.

46.- Tunkel A R, Hasbun R, Bhimraj A, Byers K, Kaplan S L, Scheld W M, et al. 2017 Infectious Diseases Society of America's clinical practice guidelines for healthcare-associated ventriculitis and meningitis. Clin Infect Dis 2017; 64 (6): e34-65. doi: 10.1093/cid/ciw861. 47.- Ranotkar S, Kumar P, Zutshi S, Prashanth K $\mathrm{S}$, Bezbaruah B, Anand J, et al. Vancomycinresistant enterococci: Troublemaker of the 21 st century. J Glob Antimicrob Resist. 2014; 2 (4):
205-12. doi: 10.1016/j.jgar.2014.04.002. 48.- Rehm S J, Tice A. Staphylococcus aureus: methicillin-susceptible $S$. aureus to methicillinresistant $S$. aureus and vancomycin-resistant S. aureus. Clin Infect Dis 2010; 51 Suppl 2:S176-S182. doi: 10.1086/653518. 